\title{
Application of a light-front coupled-cluster method to quantum electrodynamics
}

\section{Sophia CHABYSHEVA*}

University of Minnesota-Duluth, USA

E-mail: schabyshed.umn.edu

\begin{abstract}
A field-theoretic formulation of the exponential-operator technique is applied to a Hamiltonian eigenvalue problem in electrodynamics, quantized in light-front coordinates. Specifically, we consider the dressed-electron state, without positron contributions but with an unlimited number of photons, and compute its anomalous magnetic moment. A simple perturbative solution immediately yields the Schwinger result of $\alpha / 2 \pi$. The nonperturbative solution, which requires numerical techniques, sums a subset of corrections to all orders in $\alpha$ and incorporates additional physics.
\end{abstract}

Sixth International Conference on Quarks and Nuclear Physics

April 16-20, 2012

Ecole Polytechnique, Palaiseau, Paris

\footnotetext{
* Speaker.

${ }^{\dagger}$ This work was done in collaboration with J.R. Hiller and supported in part by the US Department of Energy and the Minnesota Supercomputing Institute.
} 


\section{Introduction}

Although the nonperturbative light-front coupled-cluster (LFCC) method [1] is intended for strongly coupled theories, where perturbation theory is of limited use, we explore its utility in the context of a gauge theory by considering the dressed-electron state in quantum electrodynamics (QED) [2]. The method requires the light-front coordinates of Dirac [3, 4], where the Hamiltonian evolves a state along the time direction $x^{+}=t+z$. The spatial coordinates are $\underline{x}=\left(x^{-} \equiv t-z, \vec{x}_{\perp} \equiv\right.$ $(x, y))$. The light-front energy conjugate to the chosen time is $p^{-} \equiv E-p_{z}$, and the corresponding light-front momentum is $\underline{p}=\left(p^{+} \equiv E+p_{z}, \vec{p}_{\perp} \equiv\left(p_{x}, p_{y}\right)\right)$. In these coordinates, the fundamental Hamiltonian eigenvalue problem is $\mathscr{P}^{-}|\psi\rangle=\frac{M^{2}+P_{\perp}^{2}}{P^{+}}|\psi\rangle$. Ordinarily, this eigenvalue problem is solved approximately by a truncated Fock-space expansion of the eigenstate. The LFCC method solves the problem without Fock-space truncation by building the eigenstate as $|\psi\rangle=\sqrt{Z} e^{T}|\phi\rangle$ from a valence state $|\phi\rangle$ and an operator $T$ that increases particle number while conserving any quantum numbers of the valence state. The constant $Z$ is a normalization factor.

The valence state is then an eigenstate of an effective Hamiltonian $\overline{\mathscr{P}^{-}}=e^{-T} \mathscr{P}^{-} e^{T}$, which can be computed using a Baker-Hausdorff expansion $\overline{\mathscr{P}-}=\mathscr{P}^{-}+\left[\mathscr{P}^{-}, T\right]+\frac{1}{2}\left[\left[\mathscr{P}^{-}, T\right], T\right]+$ ... The new eigenvalue problem is $\overline{\mathscr{P}}-|\phi\rangle=\frac{M^{2}+P_{\perp}^{2}}{P^{+}}|\phi\rangle$. When projected onto the valence and orthogonal sectors, it becomes

$$
P_{v} \overline{\mathscr{P}}-|\phi\rangle=\frac{M^{2}+P_{\perp}^{2}}{P^{+}}|\phi\rangle, \quad\left(1-P_{v}\right) \overline{\mathscr{P}-}|\phi\rangle=0
$$

where $P_{v}$ is the projection onto the valence sector.

A matrix element such as $\left\langle\psi_{2}|\hat{O}| \psi_{1}\right\rangle$ can be calculated, with $\left|\psi_{i}\right\rangle=\sqrt{Z_{i}} e^{T_{i}}\left|\phi_{i}\right\rangle$ and $Z_{i}=$ $1 /\left\langle\phi_{i}\left|e^{T_{i}^{\dagger}} e^{T_{i}}\right| \phi_{i}\right\rangle$. We define $\overline{O_{i}}=e^{-T_{i}} \hat{O} e^{T_{i}}=\hat{O}_{i}+\left[\hat{O}_{i}, T\right]+\frac{1}{2}\left[\left[\hat{O}_{i}, T\right], T\right]+\cdots$ and, to avoid the infinite sum in the denominator,

$$
\left\langle\tilde{\psi}_{i}\right| \equiv\langle\phi| \frac{e^{T_{i}^{\dagger}} e_{i}^{T}}{\left\langle\phi\left|e^{T_{i}^{\dagger}} e^{T_{i}}\right| \phi\right\rangle}=Z_{i}\langle\phi| e^{T_{i}^{\dagger}} e^{T_{i}}=\sqrt{Z_{i}}\left\langle\psi_{i}\right| e^{T_{i}} .
$$

We then have

$$
\left\langle\psi_{2}|\hat{O}| \psi_{1}\right\rangle=\sqrt{Z_{1} / Z_{2}}\left\langle\tilde{\psi}_{2}\left|\overline{O_{2}} e^{-T_{2}} e^{T_{1}}\right| \phi_{1}\right\rangle=\sqrt{Z_{2} / Z_{1}}\left\langle\tilde{\psi}_{1}\left|\overline{O_{1}^{\dagger}} e^{-T_{1}} e^{T_{2}}\right| \phi_{2}\right\rangle^{*}
$$

and, therefore,

$$
\left\langle\psi_{2}|\hat{O}| \psi_{1}\right\rangle=\sqrt{\left\langle\tilde{\psi}_{2}\left|\overline{O_{2}} e^{-T_{2}} e^{T_{1}}\right| \phi_{1}\right\rangle\left\langle\tilde{\psi}_{1}\left|\overline{O_{1}^{\dagger}} e^{-T_{1}} e^{T_{2}}\right| \phi_{2}\right\rangle^{*}} .
$$

In the diagonal case, this reduces to

$$
\langle\psi|\hat{O}| \psi\rangle=\langle\tilde{\psi}|\bar{O}| \phi\rangle
$$

The $\left\langle\tilde{\psi}_{i}\right|$ can be shown to be left eigenstates of the effective Hamiltonian.

We apply this to QED in an arbitrary covariant gauge, for which the Pauli-Villars-regulated Lagrangian is [5]

$$
\begin{aligned}
\mathscr{L}= & \sum_{i=0}^{2}(-1)^{i}\left[-\frac{1}{4} F_{i}^{\mu v} F_{i, \mu v}+\frac{1}{2} \mu_{i}^{2} A_{i}^{\mu} A_{i \mu}-\frac{1}{2} \zeta\left(\partial^{\mu} A_{i \mu}\right)^{2}\right] \\
& +\sum_{i=0}^{2}(-1)^{i} \bar{\psi}_{i}\left(i \gamma^{\mu} \partial_{\mu}-m_{i}\right) \psi_{i}-e \bar{\psi} \gamma^{\mu} \psi A_{\mu} .
\end{aligned}
$$


Here the fundamental physical $(i=0)$ and Pauli-Villars $(i=1)$ fields appear in null combinations

$$
\psi=\sum_{i=0}^{2} \sqrt{\beta_{i}} \psi_{i}, A_{\mu}=\sum_{i=0}^{2} \sqrt{\xi_{i}} A_{i \mu}, \quad F_{i \mu \nu}=\partial_{\mu} A_{i v}-\partial_{v} A_{i \mu} .
$$

The coupling coefficients $\xi_{i}$ and $\beta_{i}$ are constrained by

$$
\xi_{0}=1, \sum_{i=0}^{2}(-1)^{i} \xi_{i}=0, \beta_{0}=1, \sum_{i=0}^{2}(-1)^{i} \beta_{i}=0 .
$$

To fix $\xi_{2}$ and $\beta_{2}$, we require chiral symmetry restoration in the zero-mass limit [6] and a zero photon mass [7]. The light-front Hamiltonian, without antifermion terms, is then found to be [5]

$$
\begin{array}{r}
\mathscr{P}^{-}=\sum_{i s} \int d \underline{p} \frac{m_{i}^{2}+p_{\perp}^{2}}{p^{+}}(-1)^{i} b_{i s}^{\dagger}(\underline{p}) b_{i s}(\underline{p})+\sum_{l \lambda} \int d \underline{k} \frac{\mu_{l \lambda}^{2}+k_{\perp}^{2}}{k^{+}}(-1)^{l} \varepsilon^{\lambda} a_{l \lambda}^{\dagger}(\underline{k}) a_{l \lambda}(\underline{k}) \\
+\sum_{i j l \sigma s \lambda} \int d y d \vec{k}_{\perp} \int \frac{d \underline{p}}{\sqrt{16 \pi^{3} p^{+}}}\left\{h_{i j l}^{\sigma s \lambda}\left(y, \vec{k}_{\perp}\right) a_{l \lambda}^{\dagger}\left(y, \vec{k}_{\perp} ; \underline{p}\right) b_{j s}^{\dagger}\left(1-y,-\vec{k}_{\perp} ; \underline{p}\right) b_{i \sigma}(\underline{p})\right. \\
\left.+h_{i j l}^{\sigma s \lambda *}\left(y, \vec{k}_{\perp}\right) b_{i \sigma}^{\dagger}(\underline{p}) b_{j s}\left(1-y,-\vec{k}_{\perp} ; \underline{p}\right) a_{l \lambda}\left(y, \vec{k}_{\perp} ; \underline{p}\right)\right\}
\end{array}
$$

with $\varepsilon^{\lambda}=(-1,1,1,1)$ and the $h_{i j l}^{\sigma s \lambda}$ known vertex functions.

\section{The dressed-electron state}

The right and left-hand valence states ( $\overline{\mathscr{P}-}$ is not Hermitian!) are $\left|\phi_{a}^{ \pm}\right\rangle=\sum_{i} z_{a i} b_{i \pm}^{\dagger}(\underline{P})|0\rangle$ and $\left\langle\tilde{\phi}_{a}^{ \pm}\right|=\langle 0| \sum_{i} \tilde{z}_{a i} b_{i \pm}(\underline{P})$. We approximate the $T$ operator with the simplest form

$$
\begin{gathered}
T=\sum_{i j l s \sigma \lambda} \int d y d \vec{k}_{\perp} \int \frac{d \underline{\underline{p}}}{\sqrt{16 \pi^{3}}} \sqrt{p^{+}} t_{i j l}^{\sigma s \lambda}\left(y, \vec{k}_{\perp}\right) a_{l \lambda}^{\dagger}\left(y p^{+}, y \vec{p}_{\perp}+\vec{k}_{\perp}\right) \\
\times b_{j s}^{\dagger}\left((1-y) p^{+},(1-y) \vec{p}_{\perp}-\vec{k}_{\perp}\right) b_{i \sigma}(\underline{p}) .
\end{gathered}
$$

The effective Hamiltonian $\overline{\mathscr{P}^{-}}$can then be constructed [2]. From this effective Hamiltonian, the right and left-hand valence-sector equations become, for $a=0,1$,

$$
m_{i}^{2} z_{a i}^{ \pm}+\sum_{j} I_{i j} z_{a j}^{ \pm}=M_{a}^{2} z_{a i}^{ \pm} \quad \text { and } \quad m_{i}^{2} z_{a i}^{ \pm}+\sum_{j}(-1)^{i+j} I_{j i} z_{a j}^{ \pm}=M_{a}^{2} z_{a i}^{ \pm}
$$

with $M_{a}$ the $a$ th eigenmass and the self-energy given by

$$
I_{j i}=(-1)^{i} \sum_{i^{\prime} l s \lambda}(-1)^{i^{\prime}+l} \varepsilon^{\lambda} \int \frac{d y d \vec{k}_{\perp}^{\prime}}{16 \pi^{3}} h_{j i^{\prime} l}^{\sigma s \lambda *}\left(y, \vec{k}_{\perp}\right) t_{i i^{\prime} l}^{\sigma s \lambda}\left(y, \vec{k}_{\perp}\right) .
$$

The valence eigenvectors are orthonormal and complete in the following sense:

$$
\sum_{i}(-1)^{i} \tilde{z}_{a i}^{ \pm} z_{b i}^{ \pm}=(-1)^{a} \delta_{a b} \text { and } \sum_{a}(-1)^{a} z_{i a}^{ \pm} \tilde{z}_{j a}^{ \pm}=(-1)^{i} \delta_{i j}
$$


The $t$ functions satisfy the projection of the effective eigenvalue problem onto one-electron/onephoton states, orthogonal to $|\phi\rangle$, which gives [2]

$$
\begin{aligned}
\sum_{i}(-1)^{i} z_{a i}^{ \pm}\left\{h_{i j l}^{ \pm s \lambda}\left(y, \vec{k}_{\perp}\right)+\right. & \frac{1}{2} V_{i j l}^{ \pm s \lambda}\left(y, \vec{k}_{\perp}\right)+\left[\frac{m_{j}^{2}+k_{\perp}^{2}}{1-y}+\frac{\mu_{l \lambda}^{2}+k_{\perp}^{2}}{y}-m_{i}^{2}\right] t_{i j l}^{ \pm s \lambda}\left(y, \vec{k}_{\perp}\right) \\
& \left.+\frac{1}{2} \sum_{i^{\prime}} \frac{I_{j i^{\prime}}}{1-y} t_{i i^{\prime} l}^{ \pm s \lambda}\left(y, \vec{k}_{\perp}\right)-\sum_{j^{\prime}}(-1)^{i+j^{\prime}} t_{j^{\prime} j l}^{ \pm s \lambda}\left(y, \vec{k}_{\perp}\right) I_{j^{\prime} i}\right\}=0,
\end{aligned}
$$

with the vertex correction

$$
\begin{aligned}
V_{i j l}^{\sigma s \lambda}\left(y, \vec{k}_{\perp}\right)= & \sum_{i^{\prime} j^{\prime} l^{\prime} \sigma^{\prime} s \lambda^{\prime}}(-1)^{i^{\prime}+j^{\prime}+l^{\prime}} \varepsilon^{\lambda^{\prime}} \int \frac{d y^{\prime} d \vec{k}_{\perp}^{\prime}}{16 \pi^{3}} \frac{\theta\left(1-y-y^{\prime}\right)}{\left(1-y^{\prime}\right)^{1 / 2}(1-y)^{3 / 2}} \\
& \times h_{j j^{\prime} l^{\prime} l^{\prime} *}^{s \lambda^{\prime} *}\left(\frac{y^{\prime}}{1-y}, \vec{k}_{\perp}^{\prime}+\frac{y^{\prime}}{1-y} \vec{k}_{\perp}\right) t_{i^{\prime} j^{\prime} l}^{\sigma^{\prime} s^{\prime} \lambda}\left(\frac{y}{1-y^{\prime}}, \vec{k}_{\perp}+\frac{y}{1-y^{\prime}} \vec{k}_{\perp}^{\prime}\right) t_{i i^{\prime} l^{\prime}}^{\sigma \sigma^{\prime} \lambda^{\prime}}\left(y^{\prime}, \vec{k}_{\perp}^{\prime}\right) .
\end{aligned}
$$

To partially diagonalize in flavor, we define $C_{a b l}^{ \pm s \lambda}\left(y, \vec{k}_{\perp}\right)=\sum_{i j}(-1)^{i+j} z_{a i}^{ \pm} z_{b j}^{ \pm} t_{i j l}^{ \pm s \lambda}\left(y, \vec{k}_{\perp}\right)$. With analogous definitions for $H, I$, and $V$, we have

$$
\begin{aligned}
& {\left[M_{a}^{2}-\frac{M_{b}^{2}+k_{\perp}^{2}}{1-y}-\frac{\mu_{l \lambda}^{2}+k_{\perp}^{2}}{y}\right] C_{a b l}^{ \pm s \lambda}\left(y, \vec{k}_{\perp}\right)} \\
& =H_{a b l}^{ \pm s \lambda}\left(y, \vec{k}_{\perp}\right)+\frac{1}{2}\left[V_{a b l}^{ \pm s \lambda}\left(y, \vec{k}_{\perp}\right)-\sum_{b^{\prime}} \frac{I_{b b^{\prime}}}{1-y} C_{a b^{\prime} l}^{ \pm s \lambda}\left(y, \vec{k}_{\perp}\right)\right]
\end{aligned}
$$

to be solved simultaneously with the valence sector equations, which depend on $C / t$ through the self-energy matrix $I$. Notice that the physical mass $M_{b}$ has replaced the bare mass in the kinetic energy term, without any need for sector-dependent renormalization [8].

In order to compute matrix elements, such as appear in the computation of form factors, we need the left-hand eigenstate. The dual to $\langle\tilde{\psi}|=\sqrt{Z}\langle\psi| e^{T}$ is a right eigenstate of $\overline{\mathscr{P}}^{\dagger}$

$$
\left|\widetilde{\psi}_{a}^{\sigma}(\underline{P})\right\rangle=\left|\widetilde{\phi}_{a}^{\sigma}(\underline{P})\right\rangle+\sum_{j l s \lambda} \int d y d \vec{k}_{\perp} \sqrt{\frac{P^{+}}{16 \pi^{3}}} l_{a j l}^{\sigma s \lambda}\left(y, \vec{k}_{\perp}\right) a_{l \lambda}^{\dagger}\left(y, \vec{k}_{\perp} ; \underline{P}\right) b_{j s}^{\dagger}\left(1-y,-\vec{k}_{\perp} ; \underline{P}\right)|0\rangle,
$$

The flavor-diagonal left-hand wave functions are $D_{a b l}^{ \pm s \lambda}\left(y, \vec{k}_{\perp}\right) \equiv \sum_{j}(-1)^{j} z_{b j}^{s} l_{a j l}^{ \pm s \lambda}\left(y, \vec{k}_{\perp}\right)$. They satisfy the coupled equations [2]

$$
\begin{aligned}
& {\left[M_{a}^{2}-\frac{M_{b}^{2}+k_{\perp}^{2}}{1-y}-\frac{\mu_{l \lambda}^{2}+k_{\perp}^{2}}{y}\right] D_{a b l}^{\sigma s \lambda}\left(y, \vec{k}_{\perp}\right)} \\
& =\tilde{H}_{a b l}^{\sigma s \lambda}\left(y, \vec{k}_{\perp}\right)+W_{a b l}^{\sigma s \lambda}\left(y, \vec{k}_{\perp}\right)-\sum_{b^{\prime}} J_{b^{\prime} a}^{\sigma} \tilde{H}_{b^{\prime} b l}^{\sigma s \lambda *}\left(y, \vec{k}_{\perp}\right),
\end{aligned}
$$

where $W_{a b l}^{\sigma s \lambda}$ is a vertex-correction analog of $V_{a b l}^{\sigma s \lambda}$, though linear in $D$, and $J_{b a}^{\sigma}$ is a self-energy analog of $I_{b a}$. Solutions for $M_{a}, z_{a i}^{\sigma}, z_{a i}^{\sigma}$, and $C_{a b l}^{\sigma s \lambda}$ are used as input. 


\section{Anomalous magnetic moment}

We compute the anomalous moment $a_{e}$ from the spin-flip matrix element [9] of the current $J^{+}=\bar{\psi} \gamma^{+} \psi$ coupled to a photon of momentum $q$ in the Drell-Yan $\left(q^{+}=0\right)$ frame [10]

$$
16 \pi^{3}\left\langle\psi_{a}^{\sigma}(\underline{P}+\underline{q})\left|J^{+}(0)\right| \psi_{a}^{ \pm}(\underline{P})\right\rangle=2 \delta_{\sigma \pm} F_{1}\left(q^{2}\right) \pm \frac{q^{1} \pm i q^{2}}{M_{a}} \delta_{\sigma \mp} F_{2}\left(q^{2}\right) .
$$

In the limit of infinite Pauli-Villars masses, and with $M_{0}=m_{e}$, the electron mass, we find [2]

$$
\begin{aligned}
F_{1}\left(q^{2}\right)=\frac{1}{\mathscr{N}}\left[1+\sum_{s} \int \frac{d y d \vec{k}_{\perp}}{16 \pi^{3}}\left\{\sum_{\lambda= \pm} l_{000}^{ \pm s \lambda *}\left(y, \vec{k}_{\perp}-y \vec{q}_{\perp}\right) t_{000}^{ \pm s \lambda}\left(y, \vec{k}_{\perp}\right)\right.\right. \\
\left.\left.-\sum_{\lambda=0}^{3} \varepsilon^{\lambda} l_{000}^{ \pm s \lambda *}\left(y, \vec{k}_{\perp}\right) t_{000}^{ \pm s \lambda}\left(y, \vec{k}_{\perp}\right)\right\}\right]
\end{aligned}
$$

and

$$
F_{2}\left(q^{2}\right)= \pm \frac{2 m_{e}}{q^{1} \pm i q^{2}} \frac{1}{\mathscr{N}} \sum_{s} \sum_{\lambda= \pm} \int \frac{d y d \vec{k}_{\perp}}{16 \pi^{3}} l_{000}^{\mp s \lambda *}\left(y, \vec{k}_{\perp}-y \vec{q}_{\perp}\right) t_{000}^{ \pm s \lambda}\left(y, \vec{k}_{\perp}\right)
$$

with

$$
\mathscr{N}=1-\sum_{s} \sum_{\lambda=0,3} \varepsilon^{\lambda} \int \frac{d y d \vec{k}_{\perp}}{16 \pi^{3}} l_{000}^{ \pm s \lambda *}\left(y, \vec{k}_{\perp}\right) t_{000}^{ \pm s \lambda}\left(y, \vec{k}_{\perp}\right)
$$

A second term is absent in $F_{2}$ because $l$ and $t$ are orthogonal for opposite spins. The $q^{2} \rightarrow 0$ limit can be taken, to find $F_{1}(0)=1$ and

$$
a_{e}=F_{2}(0)= \pm m_{e} \sum_{s \lambda} \varepsilon^{\lambda} \int \frac{d y d \vec{k}_{\perp}}{16 \pi^{3}} y l_{000}^{\mp s \lambda *}\left(y, \vec{k}_{\perp}\right)\left(\frac{\partial}{\partial k^{1}} \mp i \frac{\partial}{\partial k^{2}}\right) t_{000}^{ \pm s \lambda}\left(y, \vec{k}_{\perp}\right) .
$$

As a check, we can consider a perturbative solution

$$
t_{000}^{\sigma s \lambda}=l_{000}^{\sigma s \lambda}=h_{000}^{\sigma s \lambda} /\left[m_{e}^{2}-\frac{m_{e}^{2}+k_{\perp}^{2}}{1-y}-\frac{\mu_{l \lambda}^{2}+k_{\perp}^{2}}{y}\right] .
$$

Substitution into the expression for $a_{e}$ gives immediately the Schwinger result [11] $\alpha / 2 \pi$, in the limit of zero photon mass, for any covariant gauge.

\section{Summary}

The LFCC method provides a nonperturbative approach to bound-state problems in quantum field theories without truncation of the Fock space and without the uncanceled divergences and spectator dependence that such truncation can cause. The approximation is instead a truncation of the operator $T$ that generates contributions from higher Fock states. It is systematically improvable through the addition of more terms to $T$, with increasing numbers of particles created and annihilated.

To complete the application to the dressed-electron state, we need to solve numerically the coupled systems that determine the $t$ and $l$ functions and to use these solutions to compute the 
anomalous moment. Within the arbitrary-gauge formulation, we can test directly for gauge dependence [5]. A more complete investigation of QED would include consideration of the dressedphoton state, contributions from electron-positron pairs to the dressed-electron state, and true bound states such as muonium and positronium. These will provide some guidance for applications to quantum chromodynamics, particularly in extensions of the holographic model for mesons [12].

\section{References}

[1] S.S. Chabysheva and J.R. Hiller, A light-front coupled-cluster method for the nonperturbative solution of quantum field theories, Phys. Lett. B 711417 (2012); J.R. Hiller, A light-front coupled-cluster method for quantum field theories, contributed to these proceedings.

[2] S.S. Chabysheva and J.R. Hiller, An application of the light-front coupled-cluster method to the nonperturbative solution of QED, arXiv:1203.0250 [hep-ph].

[3] P.A.M. Dirac, Forms of relativistic dynamics, Rev. Mod. Phys. 21 (1949) 392.

[4] For reviews of light-cone quantization, see M. Burkardt, Light front quantization, Adv. Nucl. Phys. 23 (2002) 1; S.J. Brodsky, H.-C. Pauli, and S.S. Pinsky, Quantum chromodynamics and other field theories on the light cone, Phys. Rep. 301 (1998) 299.

[5] S.S. Chabysheva and J.R. Hiller, A first nonperturbative calculation in light-front QED for an arbitrary covariant gauge, Phys. Rev. D 84 (2011) 034001.

[6] S.S. Chabysheva and J.R. Hiller, Restoration of the chiral limit in Pauli-Villars-regulated light-front QED, Phys. Rev. D 79 (2009) 114017.

[7] S.S. Chabysheva and J.R. Hiller, Nonperturbative Pauli-Villars regularization of vacuum polarization in light-front QED, Phys. Rev. D 82 (2010) 034004.

[8] S.S. Chabysheva and J.R. Hiller, On the nonperturbative solution of Pauli-Villars regulated light-front QED: A comparison of the sector-dependent and standard parameterizations, Ann. Phys. 3252435 (2010).

[9] S.J. Brodsky and S.D. Drell, The Anomalous Magnetic Moment And Limits On Fermion Substructure, Phys. Rev. D 22 (1980) 2236.

[10] S.D. Drell, D.J. Levy, T.M. Yan, A field-theoretic model for electron-nucleon deep inelastic scattering, Phys. Rev. Lett. 22 (1969) 744.

[11] J. Schwinger, On quantum electrodynamics and the magnetic moment of the electron, Phys. Rev. 73, 416 (1948); Quantum electrodynamics III: The electromagnetic properties of the electronÜradiative corrections to scattering, Phys. Rev. 76, 790 (1949).

[12] G.F. de Teramond and S.J. Brodsky, Light-front holography: A first approximation to QCD, Phys. Rev. Lett. 102 (2009) 081601. 